\title{
Erratum to: Predictors of Clinical and Endoscopic Findings in Differentiating Crohn's Disease from Intestinal Tuberculosis
}

\author{
Xuefeng Li • Xiaowei Liu • Yiyou Zou • \\ Chunhui Ouyang • Xiaoping Wu • Minghuan Zhou • \\ Linlin Chen $\cdot$ Lingjuan Ye $\cdot$ Fanggen Lu
}

Published online: 9 January 2011

(C) Springer Science+Business Media, LLC 2011

Erratum to: Dig Dis Sci (2011) 56:188-196

DOI 10.1007/s10620-010-1231-4

The e-mail address of the corresponding author Faggen Lu was erroneously published as lufangyao@163.com in the paper, correct e-mail address of address of the corresponding author is lufanggenyao@.com.

The online version of the original article can be found under doi:10.1007/s10620-010-1231-4.

X. Li · X. Liu · C. Ouyang · X. Wu · L. Ye · F. Lu (ه)

Division of Digestive Disease, 2nd Xiangya hospital,

Central South University, 410011 Changsha, Hunan, China

e-mail: lufanggenyao@163.com

Y. Zou · L. Chen

Division of Digestive Disease, Xiangya Hospital,

Central South University, 410078 Changsha, Hunan, China

M. Zhou

Division of Digestive Disease, The Affiliated Hospital of

University of Jishou, 416000 Jishou, Hunan, China 